\title{
Antipsychotic use in dementia: the relationship between neuropsychiatric symptom profiles and adverse outcomes
}

\author{
Christoph Mueller ${ }^{1,2} \oplus \cdot$ Christeena John $^{1} \cdot$ Gayan Perera $^{1} \cdot$ Dag Aarsland ${ }^{1,3} \cdot$ Clive Ballard $^{1,4} \cdot$ Robert Stewart $^{1,2}$
}

Received: 28 January 2019 / Accepted: 2 May 2020 / Published online: 15 May 2020

(c) The Author(s) 2020

\begin{abstract}
Antipsychotic treatments are associated with safety concerns in people with dementia. The authors aimed to investigate whether risk of adverse outcomes related to antipsychotic prescribing differed according to major neuropsychiatric syndromes-specifically psychosis, agitation, or a combination. A cohort of 10,106 patients with a diagnosis of dementia was assembled from a large dementia care database in South East London. Neuropsychiatric symptoms closest to first dementia diagnosis were determined according to the Health of the Nation Outcome Scales' mental and behavioural problem scores and the sample was divided into four groups: 'agitation and psychosis', 'agitation, but no psychosis', 'psychosis, but no agitation', and 'neither psychosis nor agitation'. Antipsychotic prescription in a one-year window around first dementia diagnosis was ascertained as exposure variable through natural language processing from free text. Cox regression models were used to analyse associations of antipsychotic prescription with all-cause and stroke-specific mortality, emergency hospitalisation and hospitalised stroke adjusting for sixteen potential confounders including demographics, cognition, functioning, as well as physical and mental health. Only in the group 'psychosis, but no agitation' $(n=579), 30 \%$ of whom were prescribed an antipsychotic, a significant antipsychotic-associated increased risk of hospitalised stroke was present after adjustment (adjusted hazard ratio (HR) 2.16; 95\% confidence interval (CI) 1.09-4.25). An increased antipsychotic-related all-cause (adjusted HR 1.14; 95\% CI 1.04-1.24) and stroke-specific mortality risk (adjusted HR 1.28; 95\% CI 1.01-1.63) was detected in the whole sample, but no interaction between the strata and antipsychotic-related mortality. In conclusion, the adverse effects of antipsychotics in dementia are complex. Stroke risk may be highest when used in patients presenting with psychosis without agitation, indicating the need for novel interventions for this group.
\end{abstract}

Keywords Dementia $\cdot$ Antipsychotics $\cdot$ Risk stratification $\cdot$ Prognosis $\cdot$ Mortality $\cdot$ Stroke

\section{Introduction}

Electronic supplementary material The online version of this article (https://doi.org/10.1007/s10654-020-00643-2) contains supplementary material, which is available to authorized users.

Christoph Mueller

christoph.mueller@kcl.ac.uk

1 King's College London, Institute of Psychiatry, Psychology and Neuroscience, De Crespigny Park, London, UK

2 South London and Maudsley NHS Foundation Trust, London, UK

3 Stavanger University Hospital, Centre for Age-Related Disease, Stavanger, Norway

4 University of Exeter Medical School, Exeter, UK
Neuropsychiatric symptoms, such as agitation and psychosis, are common and highly impactful complications of dementia [1] and are major determinants of poor quality of life, carer burden and healthcare costs. They are also associated with more rapid dementia progression and increased mortality $[2,3]$. Antipsychotic medications are often considered in the management of these symptoms; however, while metaanalyses indicate significant benefits, these are small, with Cohen's $d$ effect sizes lower than 0.2 across trials of antipsychotics to treat psychosis in Alzheimer's disease [4-7].

These very modest benefits have to be balanced against potential harms, and concerns have been raised around an increased risk of mortality, cerebrovascular events and hastened cognitive decline in patients with dementia prescribed these agents $[8,9]$. The US Food and Drug Administration 
has issued a black box warning against the use of antipsychotic medication in dementia related psychosis and has not approved any antipsychotic medication for treatment of aggression in dementia [6]. Conversely, the European Medicines Agency has approved risperidone as the only antipsychotic for the short-term treatment of persistent aggression in patients with moderate to severe Alzheimer's dementia who are not responsive to non-pharmacological interventions and who pose a risk of harm to self or others [10].

Agitation and psychosis are nonetheless frequently interlinked in dementia [11] and there is an increasing recognition that patient characteristics, especially the presence of certain neuropsychiatric symptoms, may be strong influencers of risks related to antipsychotic prescribing [3, 12]. Using routinely collected and clinician-rated 'real-world' data on the hyperactivity and psychosis clusters of neuropsychiatric symptoms in dementia [13], we aimed to investigate whether antipsychotic medications pose different hazards for adverse health outcomes in stratified analyses, in order to evaluate the potential for a more targeted approach to antipsychotic prescribing in dementia. The presence of additional cerebral pathologies in psychosis in dementia [14-17] suggests higher antipsychotic hazards in this group, but a substantial proportion of patients with psychosis in dementia are not distressed or agitated [18]. As neuropsychiatric symptoms have been reported as potentially stronger correlates of adverse outcomes than their treatments [19] (with agitation possibly yielding a higher risk than psychosis [3]) we hypothesized that patients with psychosis only might be at an increased risk of antipsychotic-related adverse outcomes, while in other groups addressing the impact of the distress/aggression itself might partly counteract the adverse effects of antipsychotics. As secondary outcomes we evaluated whether risks differed in relation to dementia subtype diagnosis or specific antipsychotic prescribed.

\section{Methods}

\section{Data source}

Data for this study were assembled using the South London and Maudsley NHS Foundation Trust (SLaM) Clinical Record Interactive Search (CRIS) platform. SLaM serves a population of over 1.36 million residents, across four South London boroughs (Lambeth, Lewisham, Southwark, and Croydon) and is one of Europe's largest healthcare providers for dementia and mental illness. Since 2006 all services in SLaM have adopted fully-electronic health records to enhance confidential and efficient storage of information. CRIS provides research access to over 400,000 anonymized health records from SLaM within a robust governance framework $[20,21]$ and has received ethical approval as an anonymized data resource (Oxford Research Ethics Committee $\mathrm{C}$, reference 08/H0606/71+5).

We extracted data from structured fields routinely completed in the source record and from clinical documents. Identification of relevant information from free-text records was conducted through bespoke natural language (NLP) processing algorithms using the General Architecture for Text Engineering (GATE) software [22]. See supplementary document for a detailed description of the NLP algorithms applied to ascertain antipsychotic prescription, diagnosis and Mini-mental State Examination Score (MMSE) [23]. Further, CRIS has been linked to national data on hospitalisations (Hospital Episode Statistics (HES)) [24] and Office of National Statistics (ONS) death certificate data, enabling relevant health outcome data (mortality, hospitalisation) to be extracted for the current analyses.

\section{Sample and stratification}

We included patients who received a first diagnosis of dementia in SLaM services between 1st January 2007 and 31st December 2015. Patients were excluded if they had a history of psychotic disorder prior to onset of dementia (as in this population antipsychotics are prescribed for functional psychosis rather than dementia), if they were diagnosed with a Lewy body dementia (as these pathologies inherently carry higher antipsychotic risks), and if they made use of acute hospital psychiatric liaison services in a six months (3 months either side) window around dementia diagnosis (as these patients tend to have higher levels of co-morbidities and are more likely to be subject to short-term use of antipsychotics for delirium episodes).

To stratify the sample according to neuropsychiatric symptoms present at the time of dementia diagnosis, we used the Health of the Nation Outcome Scales (HoNOS) [25], whereby the score recorded closest to first dementia diagnosis was applied. The HoNOS is a validated and wellestablished measure of patient wellbeing commonly used in UK mental health and dementia services and comprises 12 clinician-rated subscales. Each subscale is rated from 0 (no problem) to 4 (severe or very severe problem). For ease of interpretation, the scores were dichotomised to 'minor or no problems' (scores 0 or 1) and 'mild to severe problems' (scores 2-4). 'Agitation' was defined on the basis of a score of at least two or more on the HoNOS 'behavioural disturbance' scale, and 'psychosis' on the basis of a score of two or more on the HoNOS 'problems associated with hallucinations and/or delusions' scale. Both items are associated with an increased all-cause mortality risk in populations of people with dementia [26, 27].

From this information, we created four groups: 'agitation and psychosis' $(\mathrm{Ag}+\mathrm{P}+)$, 'psychosis, but no agitation' 
$(\mathrm{Ag}-\mathrm{P}+)$, 'agitation, but no psychosis' $(\mathrm{Ag}+\mathrm{P}-)$, and 'neither agitation nor psychosis' (Ag-P-).

To achieve one of our secondary objectives, we stratified the sample according to dementia diagnosis subtype. The dementia subtype diagnosis was classified according to ICD-10 [28] through NLP supported by structured fields in the source record as follows: Alzheimer's disease (F00.0 and F00.1), vascular dementia (F01), mixed-type dementia (including Alzheimer's disease and vascular dementia or F00.2) and other/unspecified dementia (F03 or no subtype mentioned).

\section{Exposure and outcome variables}

GATE-supported natural language processing algorithms on CRIS include one which ascertains pharmacotherapy from a comprehensive gazetteer of all past and current generic medication names (and most trade names); this was used to identify patients recorded as taking antipsychotic medications in a one year window (6 months either side) around first dementia diagnosis.

This medications application is designed to extract the names of medications that are inferred as currently prescribed to the patient $[29,30]$ and is described in more detail in the accompanying supplementary document, including examples of text captured. The application was developed through expert annotation, whereby domain experts coded whether the medication prescription was present either based on their expert experience and/or pre-defined coding rules. Negation statements (e.g. 'not using any antipsychotic') are not specifically ascertained as a group, but are subsumed in the wider category of non-relevant statements [21]. The application preferentially detects medications with corresponding dosage information, or where present use is explicitly mentioned (e.g. 'currently on') or is discernible through inference (e.g. 'patient' is faring better on risperidone). The precision for current use of antipsychotic medication across CRIS was found to be $81 \%$ and the recall of ever use of antipsychotic medication $77 \%$.

First, all medications in chapter 4.2. (Central nervous system/Drugs used in psychoses and related disorders) of the British National Formulary [31] were considered as exposures. To examine whether specific antipsychotics differed in their hazard profile we further determined whether patients were prescribed risperidone, olanzapine, quetiapine, any second-generation antipsychotic or any first-generation antipsychotic.

In the whole cohort and aforementioned strata (neuropsychiatric symptoms/subtype diagnoses) patients receiving any antipsychotic were compared to non-receivers in relation to adverse health outcomes. We established the time to events for each patient for the following four outcomes: (1) allcause mortality, (2) any emergency hospitalisation to acute care (non-psychiatric) hospitals [24], (3) hospitalisation due to stroke, and (4) stroke-specific mortality. Hospitalised stroke was defined on the basis of ICD-10 codes [28]. As the narrow definition of stroke (ICD-10 codes I61, I63, and I64) usually applied to national hospitalisation (HES) data [32] might miss a substantial proportion of confirmed stroke cases and misclassify a considerable number of suspected strokes [33], we applied a wider definition identifying hospitalisations in which I60 to I67 (Cerebrovascular diseases section) and G45 (Transient cerebral ischaemic attacks and related syndromes) were recorded as primary diagnosis in discharge documentation. We only considered the primary diagnosis to ensure that the stroke was a new event and the main cause for the hospitalisation. A patient was considered to have died of a stroke if the aforementioned ICD-10 codes were listed as underlying or primary cause of death on ONS death certificate data. Mortality data was available to a census point on 10th December 2016 and hospitalisation data until a census point on 31st March 2016.

\section{Covariates}

From structured fields and supported by natural language processing applications we ascertained socio-demographic factors (age, gender, marital status, ethnicity, and a neighbourhood-level index of multiple deprivation [34]), level of cognitive impairment (identified via MMSE score closest to the date of diagnosis [23]), and whether the patients had been hospitalised in the year before dementia diagnosis. In addition, we extracted data from the relevant remaining HoNOS subscales.

\section{Statistical analyses}

STATA 13 [35] was used for all analyses. Descriptive statistics were generated and presented accordingly. We constructed three Cox regression models to examine whether exposure to antipsychotics was related to the adverse outcomes (hospitalisation, stroke, all-cause mortality, strokespecific mortality). Model 1 was adjusted for age and gender. Model 2 included age, gender, marital status, ethnicity, index of deprivation, MMSE score and dementia subtype (except in the analysis stratified for dementia subtype). In Model 3 the items from Model 2 were included and we added HoNOS subscales (excluding agitation and psychosis when we stratified for these variables) and previous hospitalisation.

First, we examined antipsychotic risks in the whole sample and then in the aforementioned strata (neuropsychiatric symptoms/subtype diagnoses). We further performed separate analyses for each individual subgroup (symptom profile/ subtype diagnosis), comparing the HR in the subgroup to the HR in all other subgroups combined by including an interaction term (treatment* subgroup) in Model 3. As the power of 
the test for interaction is lower compared to the test of direct effects, we followed the recommendation to raise the type one error rate to increase power [36]. We elected to increase the error rate from $5 \%$ to $10 \%$ and considered interactions with $p<0.1$ as true interactions. Lastly, again using the full sample, different antipsychotic medications were compared in relation to hazardous outcomes.

Of patients with HoNOS scores on agitation and psychosis available at dementia diagnosis, $18 \%$ had missing data on at least one of the other covariates (most commonly MMSE (10\%) or marital status (4\%) were the only missing variables). As we judged missingness in this sample to be random, we imputed missing values using chained equations to maximise statistical power [37]. Using the mi package in STATA we created 20 imputed datasets through replacing missing values through simulated values assembled from covariates and outcome values. Rubin's rules [38] were applied to combine coefficients in final analyses.

\section{Results}

We identified 14,093 patients diagnosed with dementia in SLaM services between 2007 and 2015. Of those, 1113 patients were excluded as no baseline data was available on HoNOS ratings of agitation or psychosis, 502 patients with a diagnosis of a Lewy body dementia, 645 with a previous psychotic illness and 1727 patients as they made use of liaison psychiatry services at the time of diagnosis. The final sample consisted of 10,106 patients with a mean age at diagnosis of 81.1 (SD 8.7) years. Mean MMSE score at diagnosis was 18.7 (SD 6.4), 6384 (63.2\%) patients were female and $1115(11.0 \%)$ were prescribed an antipsychotic around the time of dementia diagnosis. See Fig. 1 for a flowchart of cohort composition and outcomes.

In total 4320 (42.8\%) patients had Alzheimer's disease (AD) recorded as diagnosis, 2553 (25.3\%) had mixed-type dementia (Alzheimer's disease and vascular dementia), 1910 (18.9\%) had vascular dementia, and 1323 (13.1\%) were diagnosed as having other or unspecified dementia. Antipsychotic prescribing at the time of dementia diagnosis was most prevalent in those with other/unspecified dementia (17.9\%), followed by vascular dementia (15.4\%) and was least likely in those with Alzheimer's disease (8.4\% in pure $\mathrm{AD}$ and $8.7 \%$ in mixed-type dementia).

\section{Adverse outcomes associated with antipsychotic prescribing in the whole sample}

Of the whole cohort, $5373(53.2 \%)$ patients died in the follow-up period with a median survival time of 4.29 years (interquartile range 2.02-7.60 years). Moreover, 6797 $(67.3 \%)$ had at least one emergency hospitalisation, 670 $(6.6 \%)$ were recorded as having a hospitalised stroke and $619(6.1 \%)$ died of a stroke according to their death certificate. In Cox regression models adjusting for age, gender, ethnicity, marital status, MMSE, deprivation score and dementia subtype (Model 2), antipsychotic prescription was related to an increased risk of all-cause mortality (hazard ratio (HR) 1.22; 95\% CI 1.12-1.32) and stroke-specific mortality (HR 1.27; 95\% CI 1.02-1.60), but not for emergency hospitalisation (HR 1.07; 95\% CI 0.99-1.15) or hospitalised stroke (HR 1.02; 95\% CI 0.80-1.29). After further adjustment for HoNOS scores and previous hospitalisation (Model 3), an increased all-cause mortality risk (HR 1.14; 95\% CI 1.05-1.24) and stroke-specific mortality risk (HR 1.28; 95\% CI 1.01-1.63) remained, but no significant effects

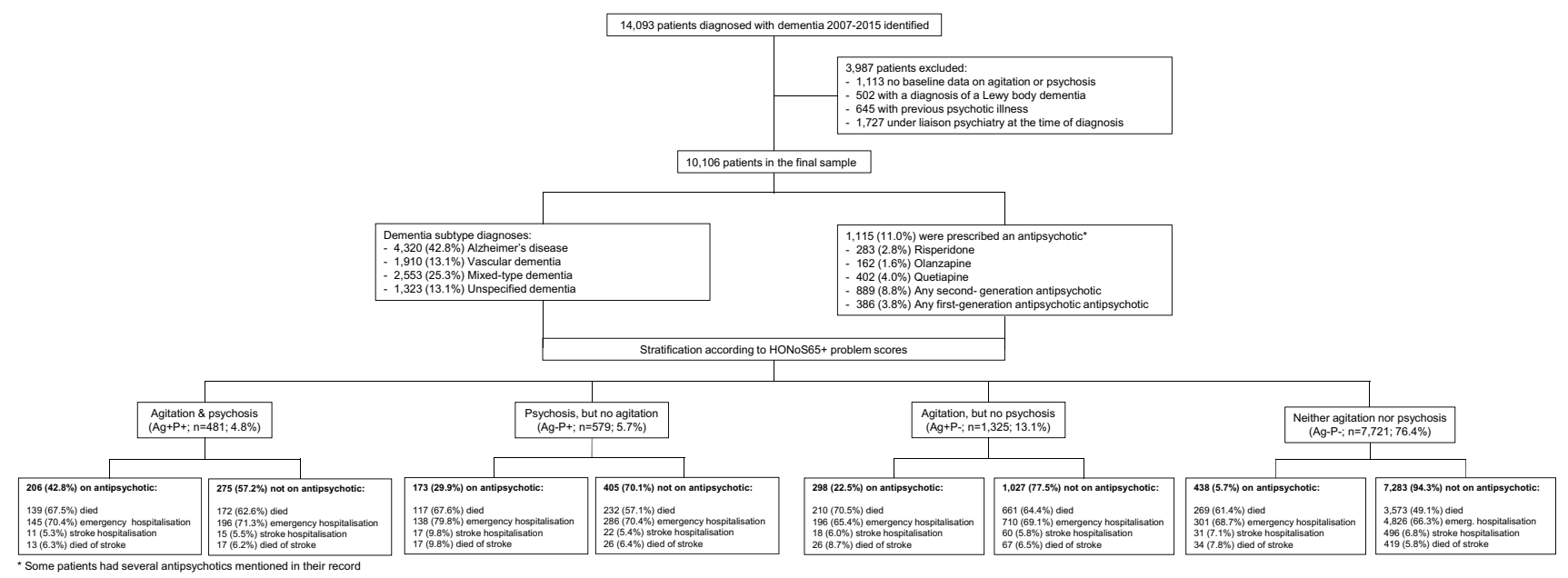

Fig. 1 Flow chart 
were detected in relation to emergency hospitalisation or stroke (see Table 4 for Model 3 and Supplementary Table 1 for results of all models).

\section{Characteristics of the sample stratified according to neuropsychiatric symptom profile}

In our sample $23.6 \%$ of patients presented with hyperactivity and psychosis neuropsychiatric symptoms at the time of dementia diagnosis; whereby agitation was present in $17.9 \%$ and psychosis in $10.5 \%$ of the sample. According to our group definitions, 481 (4.8\%) presented with 'agitation and psychosis' $(\mathrm{Ag}+\mathrm{P}+), 579$ (5.7\%) with 'psychosis, but no agitation' $(\mathrm{Ag}-\mathrm{P}+), 1325$ (13.1\%) with 'agitation, but no psychosis' (Ag+P-) and the remaining 7721 patients with neither of the two neuropsychiatric symptoms ( $\mathrm{Ag}-\mathrm{P}-$ ).
Sample characteristics and comparisons between groups are presented in Table 1. In comparison to those without either symptom, patients with agitation and/or psychosis had a lower MMSE at diagnosis, lived in more deprived neighbourhoods, were less likely to have a diagnosis of Alzheimer's disease and more likely to be diagnosed with vascular or unspecified dementia, showed an increased occurrence of depressed mood, as well as physical health and functional problems. Amongst those with neuropsychiatric symptoms, patients with 'psychosis, but no agitation' $(\mathrm{Ag}-\mathrm{P}+)$ were more likely to be female, less likely to be married or cohabiting, had a higher MMSE score, were less likely to have substance use, self-harm, physical health or functional problems (with the exception of living conditions). The $\mathrm{Ag}+\mathrm{P}+$ group included the highest proportion of patients prescribed antipsychotics (42.8\%), followed by $29.9 \%$ in the $\mathrm{Ag}-\mathrm{P}+$ group and $22.5 \%$ in $\mathrm{Ag}+\mathrm{P}-$ group.

Table 1 Sample characteristics by neuropsychiatric symptom profile

\begin{tabular}{|c|c|c|c|c|c|}
\hline Risk factors & $\begin{array}{l}\text { Agitation and } \\
\text { psychosis } \\
(\mathrm{Ag}+\mathrm{P}+) \\
(\mathrm{n}=481)\end{array}$ & $\begin{array}{l}\text { Psychosis, but no } \\
\text { agitation }(\mathrm{Ag}-\mathrm{P}+) \\
(\mathrm{n}=579)\end{array}$ & $\begin{array}{l}\text { Agitation, but no } \\
\text { psychosis }(\mathrm{Ag}+\mathrm{P}-) \\
(\mathrm{n}=1325)\end{array}$ & $\begin{array}{l}\text { Neither agitation nor } \\
\text { psychosis }(\mathrm{Ag}-\mathrm{P}-) \\
(\mathrm{n}=7721)\end{array}$ & $P$ value $^{\mathrm{a}}$ \\
\hline \multicolumn{6}{|c|}{ Socio-demographic status and cognitive function ${ }^{b}$} \\
\hline Mean age at dementia diagnosis (SD) & $81.3(10.1)$ & $81.8(9.1)$ & $81.0(9.2)$ & $81.1(8.5)$ & 0.267 \\
\hline Female gender $(\%)$ & $62.6 \% \#$ & $69.3 \% *$ & $57.6 \% *, \#$ & $63.7 \% \#$ & $<0.001$ \\
\hline Non-White ethnicity (\%) & $27.8 \%$ & $31.3 \% *$ & $23.3 \% \#$ & $24.0 \% \#$ & $<0.001$ \\
\hline Married or cohabiting status (\%) & $31.4 \%{ }^{\#}$ & $24.8 \% *$ & $34.8 \%{ }^{\#}$ & $34.9 \%{ }^{\#}$ & $<0.001$ \\
\hline Mean index of deprivation (SD) & $28.9(10.3)^{*}$ & $28.2(10.9)^{*}$ & $28.8(10.7)^{*}$ & $26.6(11.1)^{\#}$ & $<0.001$ \\
\hline Mean MMSE score at diagnosis (SD) & $15.6(7.1)^{*, \#}$ & $17.6(6.5)^{*}$ & $15.3(7.3)^{*, \#}$ & $19.5(6.0)^{\#}$ & $<0.001$ \\
\hline Dementia subtype & & & & & $<0.001$ \\
\hline Alzheimer's disease & $31.0 \% *$ & $35.1 \% *$ & $32.2 \% *$ & $45.9 \% \#$ & \\
\hline $\begin{array}{l}\text { Mixed-type dementia (including Alzhei- } \\
\text { mer's disease and Vascular dementia) }\end{array}$ & $23.7 \%$ & $24.5 \%$ & $22.9 \% *$ & $25.8 \%$ & \\
\hline Vascular dementia & $25.8 \%$ *\# & $22.5 \% *$ & $25.4 \% *$ & $17.1 \%{ }^{\#}$ & \\
\hline Unspecified or other dementia & $19.5 \% *$ & $18.0 \% *$ & $19.6 \% *$ & $11.2 \%{ }^{\#}$ & \\
\hline \multicolumn{6}{|l|}{ HoNOS symptoms/disorders $(\%)^{b}$} \\
\hline Non-accidental self-injury & $4.4 \% *$ *\# & $1.4 \%$ & $3.0 \% *$ *\# & $0.7 \%$ & $<0.001$ \\
\hline Problem-drinking or drug taking & $6.5 \% * \#$ & $2.9 \%$ & $5.4 \% *$,\# & $2.5 \%$ & $<0.001$ \\
\hline Depressed mood & $27.0 \% *, \#$ & $20.9 \% *$ & $21.9 \% *$ & $12.5 \% \#$ & $<0.001$ \\
\hline Physical illness or disability & $68.4 \% \%^{*, \#}$ & $60.8 \% *$ & $65.4 \% *$ & $47.3 \% \#$ & $<0.001$ \\
\hline \multicolumn{6}{|l|}{ HoNOS functional problems $(\%)^{b}$} \\
\hline Activities of daily living & $82.5 \%$ *,\# & $70.6 \% *$ & $81.3 \%$ *,\# & $51.6 \% \#$ & $<0.001$ \\
\hline Living conditions & $21.3 \% *$ & $18.9 \% *$ & $18.0 \% *$ & $9.3 \% \#$ & $<0.001$ \\
\hline Occupational/recreational activities & $53.5 \%$ *\# & $38.6 \% *$ & $49.9 \% *, \#$ & $27.7 \% \#$ & $<0.001$ \\
\hline Social relationships & $52.2 \%$ *,\# & $17.4 \% *$ & $45.6 \% *, \#$ & $8.3 \%$ & $<0.001$ \\
\hline Antipsychotic prescription $^{c}$ & $42.8 \%$ *,\# & $29.9 \% *$ & $22.5 \% *, \#$ & $5.7 \%^{\#}$ & $<0.001$ \\
\hline $\begin{array}{l}\text { Hospitalisation prior to dementia } \\
\text { diagnosis }^{\mathrm{d}}\end{array}$ & $52.6 \% *$ & $48.0 \%$ & $50.8 \% *$ & $46.1 \%$ & $<0.001$ \\
\hline
\end{tabular}

*Significantly different to group $\mathrm{Ag}-\mathrm{P}-(p<0.05)$; ${ }^{\#}$ significantly different to group $\mathrm{Ag}-\mathrm{P}+(p<0.05)$

${ }^{\mathrm{a}} \mathrm{ANOVA}$ or $\mathrm{Chi}^{2}$ test; ${ }^{\mathrm{b}}$ at the time of dementia diagnosis; ${ }^{\mathrm{c}}$ in a one-year window around dementia diagnosis; ${ }^{\mathrm{d}}$ in the year prior to dementia diagnosis 


\section{Hazards related to antipsychotic prescribing in the four neuropsychiatric symptom subgroups}

Multivariate Cox regression models (Model 3; see Table 2) showed a $116 \%$ and significantly increased stroke hospitalisation risk associated with antipsychotic prescribing in the $\mathrm{Ag}-\mathrm{P}+$ group, whereby no increased hazard was detected in any of the other groups.

An interaction between antipsychotic prescribing and the Ag-P + group (when comparing to the hazard in all other subgroups combined) in relation to stroke hospitalisation could further be identified ( $p=0.064$ ) strengthening the finding that antipsychotic-related risk of hospitalisation due to stroke might be higher in this group (see Supplementary Table 2).

Whereby an increased antipsychotic mortality risk was detected in a Cox regression model adjusted for age, gender, marital status, ethnicity, index of deprivation, MMSE score, and dementia subtype (Model 2) in the $\mathrm{Ag}+\mathrm{P}-$ and the $\mathrm{Ag}-\mathrm{P}$ - group, this was no longer significant after further adjustment (Model 3). No increased risks of emergency hospitalisation or stroke-specific mortality were detected in relation to any of the groups in adjusted models (Model 2 and 3).

This was mirrored in non-significant interaction terms for the association between strata and antipsychotic prescribing in relation to the adverse health outcomes (see Supplementary Table 2). Although a 20\% increased risk of emergency hospitalisation in the $\mathrm{Ag}-\mathrm{P}+$ group only amounted to a non-significant trend $(p=0.096)$, the interaction term was significant $(p=0.042)$ indicating that antipsychotic-related emergency hospitalisation risk might be higher in patients with this symptom profile than in the remainder of the sample. The absence of evidence for an increased antipsychoticrelated all-cause mortality risk by neuropsychiatric symptom strata was reflected the $p$ values of the interaction terms. A significantly increased antipsychotic-related all-cause mortality risk was identified for those not in the $\mathrm{A}+\mathrm{P}+$ group. Together with $p$ value of 0.076 for the interaction term this indicates that there might be a lower antipsychotic-related all-cause mortality risk in patients who don't suffer from co-morbid agitation and psychosis.

\section{Hazards related to antipsychotic prescribing in the four dementia subtype groups}

Multivariable Cox proportionate hazard models adjusted for age, gender, ethnicity, deprivation and MMSE (Model 2; see Table 3) showed an increased antipsychotic-related all-cause mortality and hospitalisation risk in patients diagnosed with Alzheimer's disease, as well as an increased antipsychoticrelated all-cause mortality risk in patients diagnosed with vascular dementia. After further adjustments for previous hospitalisation and HoNOS scores (including agitation and

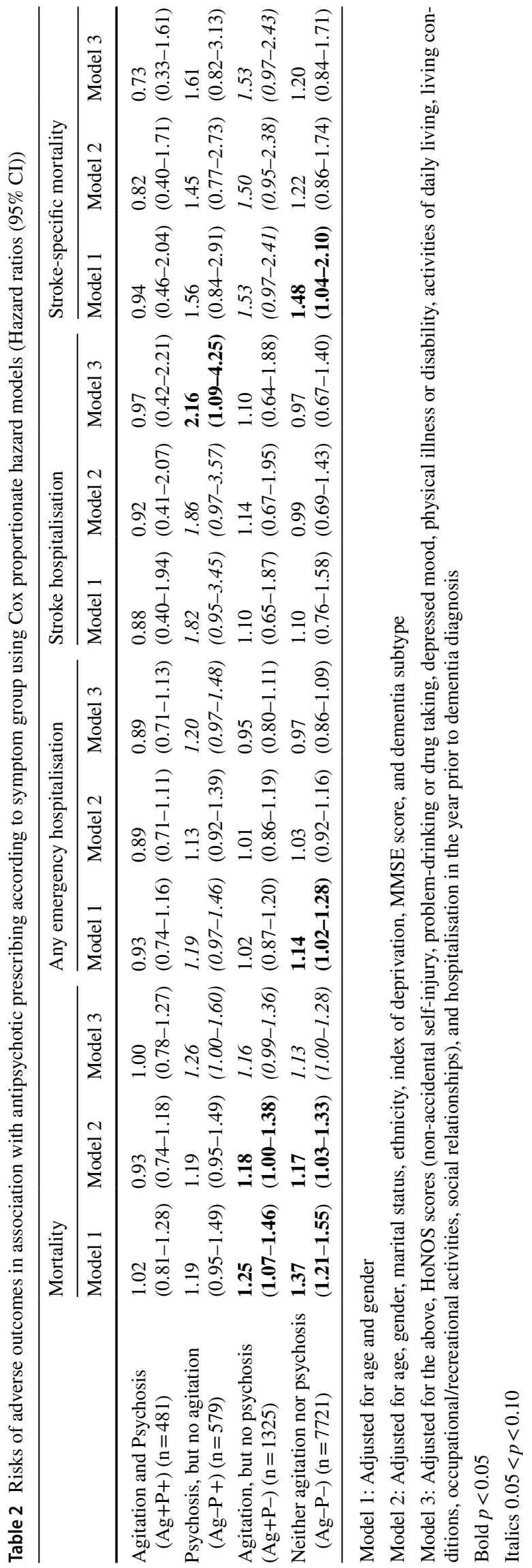


psychosis) a significantly increased antipsychotic-related mortality risk remained in patients diagnosed with Alzheimer's disease (22\% increase) and vascular dementia (29\% increase). Further, patients with mixed-type dementia had a $65 \%$ and significantly increased risk of stroke hospitalisation associated with antipsychotic prescribing.

When testing for interactions (Supplementary Table 3), the increased antipsychotic related all-cause mortality risk was strengthened in the vascular dementia group $(p=0.090)$, but not in the Alzheimer's disease group $(p=0.571)$. A significantly increased antipsychotic-related all-cause mortality risk was identified for those not in the mixed-type dementia group. Together with $p$ value of 0.001 for the interaction term this indicates that there might be a lower antipsychoticrelated all-cause mortality risk in patients with mixed-type dementia. For none of the other analyses $p$ values for interaction $<0.1$ were identified, but the there was an indication $(p=0.130)$ that the increased antipsychotic-related stroke risk in those diagnosed with mixed-type dementia might be true.

\section{Comparison of specific antipsychotics in relation to adverse outcomes}

Cox proportionate hazard models evaluating specific antipsychotic medications against no antipsychotic or any other antipsychotic are presented in Table 4 (only Model 3 ) and Supplementary Table 1 (Models 1, 2, 3). Whilst, as aforementioned, an increased all-cause and stroke-specific mortality risk was identified for use of any antipsychotic compared to non-use, this could only be replicated with significant findings for second generation antipsychotics ( $15 \%$ increased all-cause mortality risk and $31 \%$ increased stroke-specific mortality risk compared to no use of an antipsychotic). Largest all-cause mortality risk increase appeared to be associated with risperidone, although this amounted to non-significant trend $(p=0.062)$. Direct comparisons in subgroups of antipsychotic users didn't detect any significant differences between users of specific antipsychotics and users of any other antipsychotic; neither did the comparison between first- and second-generation antipsychotics.

\section{Discussion}

In a large naturalistic sample of patients diagnosed in a specialist service for dementia and mental health care, we found that patients with dementia rated by clinicians to have problematic psychosis, but not co-morbid agitation or agitation alone, were at an increased risk of stroke associated with antipsychotic use. 
Table 4 Risks of adverse outcomes according to specific antipsychotic type using Cox proportional hazard models (Hazard ratios (95\% CI))

\begin{tabular}{|c|c|c|c|c|}
\hline & Mortality & $\begin{array}{l}\text { Any emer- } \\
\text { gency hospi- } \\
\text { talisation }\end{array}$ & Stroke hospitalisation & Stroke-specific mortality \\
\hline $\begin{array}{l}\text { Any antipsychotic }(\mathrm{n}=1115) \text { versus no antipsychotic } \\
(\mathrm{n}=8991)\end{array}$ & $\begin{array}{l}1.14 \\
(1.04-1.24)\end{array}$ & $\begin{array}{l}0.99 \\
(0.92-1.08)\end{array}$ & $\begin{array}{l}1.09 \\
(0.84-1.40)\end{array}$ & $\begin{array}{l}1.28 \\
(1.01-1.63)\end{array}$ \\
\hline $\begin{array}{l}\text { Risperidone }(\mathrm{n}=283) \text { versus any other antipsychotic } \\
\quad(\mathrm{n}=832)\end{array}$ & $\begin{array}{l}1.03 \\
(0.86-1.22)\end{array}$ & $\begin{array}{l}1.00 \\
(0.84-1.18)\end{array}$ & $\begin{array}{l}0.82 \\
(0.46-1.44)\end{array}$ & $\begin{array}{l}1.07 \\
(0.65-1.75)\end{array}$ \\
\hline Risperidone $(n=283)$ versus no antipsychotic $(n=8991)$ & $\begin{array}{l}1.16 \\
(0.99-1.36)\end{array}$ & $\begin{array}{l}0.99 \\
(0.85-1.15)\end{array}$ & $\begin{array}{l}0.93 \\
(0.55-1.57)\end{array}$ & $\begin{array}{l}1.34 \\
(0.85-2.11)\end{array}$ \\
\hline $\begin{array}{l}\text { Olanzapine }(n=162) \text { versus any other antipsychotic } \\
(\mathrm{n}=953)\end{array}$ & $\begin{array}{l}1.02 \\
(0.81-1.29)\end{array}$ & $\begin{array}{l}0.81 \\
(0.65-1.00)\end{array}$ & $\begin{array}{l}0.77 \\
(0.36-1.61)\end{array}$ & $\begin{array}{l}1.27 \\
(0.68-2.35)\end{array}$ \\
\hline Olanzapine $(\mathrm{n}=162)$ versus no antipsychotic $(8991)$ & $\begin{array}{l}1.16 \\
(0.93-1.45)\end{array}$ & $\begin{array}{l}0.83 \\
(0.67-1.01)\end{array}$ & $\begin{array}{l}0.86 \\
(0.42-1.75)\end{array}$ & $\begin{array}{l}1.58 \\
(0.88-2.83)\end{array}$ \\
\hline $\begin{array}{l}\text { Quetiapine }(n=402) \text { versus any other antipsychotic } \\
(n=713)\end{array}$ & $\begin{array}{l}0.95 \\
(0.82-1.10)\end{array}$ & $\begin{array}{l}1.12 \\
(0.97-1.30)\end{array}$ & $\begin{array}{l}1.38 \\
(0.88-2.16)\end{array}$ & $\begin{array}{l}0.92 \\
(0.60-1.41)\end{array}$ \\
\hline Quetiapine $(n=402)$ versus no antipsychotic $(n=8991)$ & $\begin{array}{l}1.11 \\
(0.98-1.25)\end{array}$ & $\begin{array}{l}1.07 \\
(0.95-1.20)\end{array}$ & $\begin{array}{l}1.30 \\
(0.92-1.84)\end{array}$ & $\begin{array}{l}1.22 \\
(0.86-1.73)\end{array}$ \\
\hline $\begin{array}{l}\text { Any second-generation }(n=889) \text { antipsychotic versus no } \\
\text { antipsychotic }(n=8991)\end{array}$ & $\begin{array}{l}1.15 \\
(1.05-1.26)\end{array}$ & $\begin{array}{l}1.02 \\
(0.93-1.11)\end{array}$ & $\begin{array}{l}1.13 \\
(0.86-1.49)\end{array}$ & $\begin{array}{l}1.31 \\
(1.00-1.70)\end{array}$ \\
\hline $\begin{array}{l}\text { Any first-generation }(\mathrm{n}=386) \text { antipsychotic versus no } \\
\text { antipsychotic }(\mathrm{n}=8991)\end{array}$ & $\begin{array}{l}1.10 \\
(0.96-1.25)\end{array}$ & $\begin{array}{l}0.94 \\
(0.82-1.06)\end{array}$ & $\begin{array}{l}1.06 \\
(0.71-1.58)\end{array}$ & $\begin{array}{l}1.12 \\
(0.76-1.65)\end{array}$ \\
\hline $\begin{array}{l}\text { Any first-generation antipsychotic }(n=223) \text { versus any } \\
\text { second-generation }(n=762) \text { antipsychotic* }\end{array}$ & $\begin{array}{l}0.95 \\
(0.79-1.14)\end{array}$ & $\begin{array}{l}0.89 \\
(0.74-1.07)\end{array}$ & $\begin{array}{l}0.86 \\
(0.48-1.57)\end{array}$ & $\begin{array}{l}0.84 \\
(0.49-1.44)\end{array}$ \\
\hline
\end{tabular}

Adjusted for age, gender, marital status, ethnicity, index of deprivation, MMSE score, dementia subtype, HoNOS scores (agitation, psychosis, non-accidental self-injury, problem-drinking or drug taking, depressed mood, physical illness or disability, activities of daily living, living conditions, occupational/recreational activities, social relationships), and hospitalisation in the year prior to dementia diagnosis (Model 3)

Bold $p<0.05$

Italics $0.05<p<0.10$

The antipsychotic first mentioned can also be in combination (e.g. if risperidone prescribed in combination, it is grouped in the risperidone group)

* SGA versus FGA excludes combinations of second-generation with first-generation antipsychotics $(\mathrm{n}=163)$

A higher all-cause and stroke-specific mortality risk related to antipsychotic prescribing was detected in the whole sample. There was no true interaction between neuropsychiatric symptom strata and antipsychotic-related mortality risk, with the exception of a possibly higher antipsychotic-related mortality risk in those not in the agitation and psychosis group.

Further, an increased antipsychotic-related all-cause mortality risk was identified for patients with Alzheimer's disease and vascular dementia, whereby an interaction between dementia subtype strata and antipsychotic mortality risk could only be identified for vascular dementia.

In the whole sample, second-generation antipsychotics were associated with an increased all-cause and strokerelated mortality risk compared to non-use, and this association amounted to a trend for risperidone. No other specific antipsychotic was significantly associated with a higher risk of adverse outcomes, neither in comparison to all other antipsychotic users nor non-users.

Almost half of those presenting with agitation and psychosis $(\mathrm{Ag}+\mathrm{P}+)$ were prescribed antipsychotics, indicating a group in whom clinicians saw the greatest need for treatment, followed by those with psychosis alone, $30 \%$ of whom had antipsychotic treatment recorded. In our sample $24 \%$ of patients presented with neuropsychiatric symptoms at the time of dementia diagnosis, a symptom prevalence which is slightly lower than reported in previous research $[3,39]$. The likely reason for this is that we excluded symptoms classified as 'minor problem, requiring no action' on the HoNOS scale, which might be included if a structured research scale is applied and recording is likely to be less rigorous in routinely collected data than in screened research samples.

The excess stroke risk associated with antipsychotic use in the $\mathrm{Ag}-\mathrm{P}+$ group highlights challenges in the treatment of psychosis in dementia for which antipsychotics are frequently used. They are known to have at best modest efficacy in these circumstances and important side effect risks. In addition to the effects found in this study, antipsychotics are also associated with accelerated cognitive decline, sedation and extrapyramidal symptoms $[6,9,40]$.

The results of this study differ from a meta-analysis data of data from 1721 patients included in randomised controlled 
trials of risperidone [12], which found that in those with delusions at baseline (as identified on the Behavioral Pathology in Alzheimer's Disease (BEHAVE-AD) scale [41]), the risperidone-related stroke risk was not significantly increased (HR 1.47; 95\% CI 0.59-3.65) as distinct from a raised risk in those who did not present with delusions (HR 5.88; 95\% CI 2.09-16.53). Several explanations exist for these differences: While risperidone trials used a structured measure (BEHAVE-AD [41]), our classification of psychotic symptoms was according to the relevant HoNOS subscale. In this measure, the clinician rates if the neuropsychiatric symptom cluster poses a problem for the patient and their environment rather than describing the nature of the psychotic symptom. Further the inclusion criteria differed between the meta-analysis and our study. Risperidone trials [12] only included patients if they displayed psychosis or scored above a threshold on BEHAVE-AD [41], leading to a mixed population of patients with psychosis, agitation or a combination of neuropsychiatric symptoms. Our study aimed to examine agitation and psychosis as combined or mutually exclusive. We aimed to assess a class effect of antipsychotics in relation to neuropsychiatric symptom profiles and did not investigate specific properties of individual medications in relation to the strata. It should also be borne in mind that clinical trials inevitably recruit rather selected samples, whereas our cohort may be more representative of the treated population.

There are several explanations why patients with dementia presenting with psychotic symptoms might be at a higher risk of antipsychotic-related stroke. First, the presence of tau pathology has both been linked to the presence of psychotic symptoms and an adverse prognosis in Alzheimer's disease $[17,42,43]$. Evidence from mouse models suggests that tau mediates excitotoxicity after cerebrovascular events [44]. It is therefore possible that small cerebrovascular events triggered by antipsychotic-related excessive sedation, dehydration or orthostatic hypotension [45] might lead to a deficit in brain perfusion, and the response to this deficit might be exaggerated to toxic levels in the presence of tau pathology, ultimately leading to a hospitalised stroke [44]. In addition, a recent study of autopsied cases of AD demonstrated that cerebral amyloid angiopathy and advanced small vessel disease were more common in $\mathrm{AD}$ patients with psychosis than in those without psychosis [15]. Although its role in the neurodegenerative process is not fully understood, cerebral amyloid angiopathy weakens cerebral blood vessels [46] and can lead to microbleeds or larger haemorrhages. It is therefore possible that psychosis in Alzheimer's disease is related to vascular fragility, and antipsychotics might increase risk of stroke via the aforementioned mechanisms.

Moreover, there is an increasing recognition that patients diagnosed with a single dementia subtype, as Alzheimer's disease, frequently have co-morbid cerebral pathologies, as
Lewy bodies, vascular pathology, or TDP-43 proteinopathy, when autopsied [47]. The presence of Lewy bodies is associated with a higher frequency of visual hallucinations [47-49] as well as with sensitivity reactions to antipsychotic medications, and might thereby explain in part the increased risk of adverse outcomes $[16,50]$.

We didn't not find evidence for an increased antipsychotic-related stroke risk in patients with agitation, both in those with and those without co-morbid psychosis. While pathological and anatomical changes in psychosis in dementia have been extensively studied and also distinguished from psychosis in schizophrenia [51], less is known about agitation in dementia. Agitation in dementia has been associated with frontal lobe dysfunction [52] and brain regions involved in subjective emotional experiences [53]. It has been hypothesised that agitation could arise from overestimating or misinterpreting potential threats [54], whereby those threats could for example be pain or changes in the environment. It is however also conceivable that, as possibly in the agitation and psychosis group, those overinterpreted threats are psychotic or quasi psychotic experiences. Further research is required to clarify the neuropathological corelates of agitation in dementia, especially distinguishing between 'agitation as a syndrome' from 'agitation as response to another disorder' (e.g. agitation and psychosis) [52], which could also elucidate possible mechanisms for differences in antipsychotic hazards compared to those with only psychosis.

When we stratified by dementia subtype, we identified a robust $29 \%$ increased antipsychotic related mortality risk in patients diagnosed with vascular dementia. This is different from a previous study by Sultana and colleagues in this data source [30], which might be grounded in two key differences between the two studies: First, we ascertained prevalent use of antipsychotic medication at dementia diagnosis and the previous study antipsychotic use at any timepoint after dementia diagnosis. Second, Sultana and colleagues only included second-generation (atypical) antipsychotics, while our study evaluated the impact of all classes of antipsychotics and first-generation antipsychotics have recently been shown to yield a higher mortality risk in those with cardiovascular or cerebrovascular disease [55].

We could only identify a class effect for all antipsychotics and second-generation antipsychotics in relation to mortality risk. Although no significant results were obtained, there was a trend towards a higher mortality risk in those taking risperidone, which was also the largest group taking a specific antipsychotic. In line with previous research [56], Quetiapine appeared to yield lowest risk of mortality. The absence of evidence for increased hazards in the firstgeneration antipsychotic group is in line with a recent study [57] showing that patients with Alzheimer's disease using 
first-generation antipsychotics had a lower risk of death than those using second-generation antipsychotics.

Finally, although this cannot be derived from our data, the dose and antipsychotic agent used might differ between prescription for agitation and for psychosis in dementia. While clear guidance exists in the UK and Europe for cautious management of agitation $[10,58]$, prescribing for psychosis is off-label and clinicians might use higher doses leading to an increased excess risk of harm [56].

Strengths of this study include the large naturalistic sample of patients diagnosed with dementia by a near-monopoly dementia care provider for its catchment population. The use of clinician-rated real-world measures of neuropsychiatric problems should also give a clinically relevant picture. MMSE scores in our sample were higher than in most clinical trials conducted on antipsychotics in people with dementia, which largely include participants in the severe stage of illness [12]. For this reason, similar to CATIE-AD [59], our findings might be more applicable in earlier disease stages and thereby more relevant to patients and their families. The richness of this data allowed adjustment for a wide range of confounders, and the linkage to national data on mortality and hospitalisation should have resulted in close to complete ascertainment of outcome data. A particular advantage of NLP is that the applications account for the linguistic context of a statement of interest in free text, enabling exclusion of negation and other irrelevant statements and ascertaining current, rather than past, prescription or speculations about future prescribing [29]. However, the use of routinely collected electronic health record data also presents a number of limitations. First, consistent recording of patient information is only available at certain time points of the patient's journey through the healthcare system. Therefore, we chose to ascertain recording of neuropsychiatric symptoms and antipsychotic prescription in the most reliable window around the time of first dementia diagnosis. Second, we could extract information on the initiation of antipsychotic agents and on use at given times, but we were not able to track antipsychotic prescription longitudinally. However, hazardous effects of antipsychotics are already present when only prescribed short-term [60] and the agents are unlikely to be withdrawn in the absence of effective alternative interventions [61]. Nevertheless, exclusively considering exposure to antipsychotics around the time of diagnosis leads to individuals prescribed antipsychotics in a later stage of dementia not being considered as exposed, which could potentially bias estimates towards the null and is particularly relevant to the null finding in the relatively small $\mathrm{Ag}+\mathrm{P}+$ group. This group is more than 2.5 times smaller than the other groups, which might have resulted in a lack of power in adjusted analyses. Third, in addition to the aforementioned challenges with data availability and temporality further limitations of ascertaining variables through NLP from clinical records need to be acknowledged: The output depends on the accuracy and quality of data entry, which varies by individual clinician and is compromised through the use of jargon, idiosyncratic abbreviations or misspellings [21]. Although precision and recall are relatively high for the medication NLP application, there remains a risk under- or overestimating the true prescribing prevalence. Moreover, there is a possibility of misclassification of the reason for strokerelated hospitalisation or death. As we only used the first discharge diagnosis in order to ascertain new cerebrovascular events, strokes that occurred in the context of another medical event might have been missed, potentially leading to under-recording and underestimation of effects. The broader definition of the stroke outcome according to ICD-10 was used to reflect the uncertainty which often exists around cerebrovascular events in hospital settings, where distinction from TIA or other 'stroke mimics' is often not possible in the short time-frame of the admission [33], but this approach might also lead to a higher detection of false positives. That we are only detecting an increased antipsychotic-related risk of hospitalised stroke in the $\mathrm{Ag}-\mathrm{P}+$, but no increased risk of stroke-related mortality, could reflect that those patients are experience signs of stroke but not necessarily cerebrovascular changes. Conversely, death certificate data is prone to classification bias and sensitivity for recording of stroke on death certificate has been shown to be below $71 \%$ in most studies [62]. Fourth, we were only able to examine individual antipsychotics across the whole sample and did not seek to analyse by type of antipsychotic or individual agent in analyses stratified by neuropsychiatric symptom profile or subtype diagnosis. Heterogeneity by agent or sub-class can therefore not be excluded in these analyses. Fifth, physical health was ascertained through previous hospitalisation and the HoNOS 'Physical illness and disability problems' subscale. The latter, although widely used in clinical mental health services in the UK and consistently showing relations with important outcomes [26], is a relatively brief measure without detailed information on the conditions determining its score. Sixth, data on level of education was not available, and socio-economic status was only ascertained at neighbourhood rather than individual level [34]. Thus, although we were able to adjust for a range of demographic, clinical, social and functional covariates, residual confounding cannot be fully excluded. This is particularly relevant for smaller subgroups (as $\mathrm{Ag}+\mathrm{P}+$ ) where adjustment for confounders might not fully account for differences in baseline variables and correlation does not necessarily infer causality. An important consideration for pharmacoepidemiologic studies is confounding by indication, whereby individuals with more severe symptoms are more likely to be prescribed medications. We stratified patients by the presence of agitation and/or psychosis according to a well-being score but could not measure severity of these symptoms. As stroke 
prior to onset of Alzheimer's disease has been described as associated with a higher risk of delusions [14], higher levels of cerebrovascular morbidity might be present a priori in patients with psychosis prescribed an antipsychotic. Lastly, for the purpose of this study we only examined whether antipsychotic-related hazards differed in relation to agitation and psychosis as these are the two main indications for antipsychotic use in dementia. Future studies could examine whether degree of cognitive impairment, overall neuropsychiatric symptom burden or other specific neuropsychiatric symptoms (e.g. depression or anxiety) affect antipsychotic hazards, which could feed into more advanced prediction models and potentially translate into real-time risk detection and alerting systems [63].

\section{Conclusions}

Our data suggests that antipsychotic use is associated with more than a doubling of risk of cerebrovascular events in patients with dementia suffering from psychosis (without agitation). Advocacy for avoidance of antipsychotics has been strongest in for agitation in dementia [58], but this might be equally, or even more important, in those presenting with psychosis. While non-pharmacological management strategies of psychosis are becoming more accepted in the field of functional psychotic disorders [64], there is little evidence available on the efficacy of such interventions in dementia [65] and novel strategies to address distressing psychotic symptoms in dementia clearly need further development and evaluation.

Acknowledgements We would like to thank Jessica Irving, Jaya Chaturvedi, Angus Roberts and Matthew Broadbent for support with the compilation of the natural language processing supplement and Hitesh Shetty for assistance for with data extraction. We are grateful to the anonymous reviewer for their helpful comments and suggestions.

Authors contribution $\mathrm{CM}$ and $\mathrm{CJ}$ conceived the study. CM, CJ and GP performed the analysis. CM, GP and RS interpreted the data. CM, $\mathrm{CJ}$ and $\mathrm{CB}$ wrote the manuscript. DA, CB, RS provided expertise and feedback. All authors critically revised the manuscript and approved the final version of the study. This project was conducted as CJ's dissertation in the MSc in Mental Health Sciences Studies at King's College London.

Funding CM, GP, DA and RS receive salary support from the National Institute for Health Research (NIHR) Biomedical Research Centre at South London and Maudsley NHS Foundation Trust and King's College London, and RS is a NIHR Senior Investigator. The views expressed are those of the author(s) and not necessarily those of the NHS, the NIHR or the Department of Health and Social Care.

\section{Compliance with ethical standards}

Conflict of interest RS has received research funding from Roche, Pfizer, Janssen, Lundbeck and In-Silico-Bioscience. DA has received research support and/or honoraria from Astra-Zeneca, H. Lundbeck, Novartis Pharmaceuticals and GE Health, and serves as paid consultant for $\mathrm{H}$. Lundbeck and Axovant. $\mathrm{CB}$ has received honoraria and grant funding from Acadia pharmaceuticals, Lundbeck, Takeda and Axovant pharmaceutical companies. CB leads the Alzhiemer's disease psychosis (ADP) investigators group. Honoraria from Lundbeck, Lilly, Otusaka and Orion pharmaceutical companies. CM, CJ and GP declare no conflict of interest.

Open Access This article is licensed under a Creative Commons Attribution 4.0 International License, which permits use, sharing, adaptation, distribution and reproduction in any medium or format, as long as you give appropriate credit to the original author(s) and the source, provide a link to the Creative Commons licence, and indicate if changes were made. The images or other third party material in this article are included in the article's Creative Commons licence, unless indicated otherwise in a credit line to the material. If material is not included in the article's Creative Commons licence and your intended use is not permitted by statutory regulation or exceeds the permitted use, you will need to obtain permission directly from the copyright holder. To view a copy of this licence, visit http://creativecommons.org/licenses/by/4.0/.

\section{References}

1. Zhao QF, Tan L, Wang HF, et al. The prevalence of neuropsychiatric symptoms in Alzheimer's disease: systematic review and meta-analysis. J Affect Disord. 2016;190:264-71. https://doi. org/10.1016/j.jad.2015.09.069.

2. Okura T, Plassman BL, Steffens DC, Llewellyn DJ, Potter GG, Langa KM. Neuropsychiatric symptoms and the risk of institutionalization and death: the aging, demographics, and memory study. J Am Geriatr Soc. 2011;59(3):473-81. https://doi.org/10.1 111/j.1532-5415.2011.03314.x.

3. Peters ME, Schwartz S, Han D, et al. Neuropsychiatric symptoms as predictors of progression to severe Alzheimer's dementia and death: the Cache County Dementia Progression Study. Am J Psychiatry. 2015;172(5):460-5. https://doi.org/10.1176/appi. ajp.2014.14040480.

4. Seitz DP, Adunuri N, Gill SS, Gruneir A, Herrmann N, Rochon P. Antidepressants for agitation and psychosis in dementia. Cochrane Database Syst Rev. 2011;2:CD008191. https://doi. org/10.1002/14651858.cd008191.pub2.

5. Ballard C, Corbett A. Agitation and aggression in people with Alzheimer's disease. Curr Opin Psychiatr. 2013;26(3):252-9. https://doi.org/10.1097/YCO.0b013e32835f414b.

6. Maher AR, Maglione M, Bagley S, et al. Efficacy and comparative effectiveness of atypical antipsychotic medications for off-label uses in adults: a systematic review and meta-analysis. JAMA. 2011;306(12):1359-69. https://doi.org/10.1001/jama.2011.1360.

7. Schneider LS, Dagerman K, Insel PS. Efficacy and adverse effects of atypical antipsychotics for dementia: meta-analysis of randomized, placebo-controlled trials. Am J Geriatr Psychiatr. 2006;14(3):191-210. https://doi.org/10.1097/01.JGP.0000200589 $.01396 .6 \mathrm{~d}$.

8. Schneider LS, Tariot PN, Dagerman KS, et al. Effectiveness of atypical antipsychotic drugs in patients with Alzheimer's disease. N Engl J Med. 2006;355(15):1525-38. https://doi.org/10.1056/ NEJMoa061240. 
9. Tampi RR, Tampi DJ, Balachandran S, Srinivasan S. Antipsychotic use in dementia: a systematic review of benefits and risks from meta-analyses. Ther Adv Chron Dis. 2016;7(5):229-45. https://doi.org/10.1177/2040622316658463.

10. European Medicines Agency. Risperdal. 2008. http://www.ema. europa.eu/ema/index.jsp?curl=pages/medicines/human/referrals/ Risperdal/human_referral_000022.jsp\&mid=WC0b01ac05805c5 16f. Accessed 20th April 2018.

11. Shub D, Ball V, Abbas AA, Gottumukkala A, Kunik ME. The link between psychosis and aggression in persons with dementia: a systematic review. Psychiatr Q. 2010;81(2):97-110. https://doi. org/10.1007/s11126-009-9121-7.

12. Howard R, Costafreda SG, Karcher K, Coppola D, Berlin JA, Hough D. Baseline characteristics and treatment-emergent risk factors associated with cerebrovascular event and death with risperidone in dementia patients. Br J Psychiatr. 2016;209(5):378-84. https://doi.org/10.1192/bjp.bp.115.177683.

13. Petrovic M, Hurt C, Collins D, et al. Clustering of behavioural and psychological symptoms in dementia (BPSD): a European Alzheimer's disease consortium (EADC) study. Acta Clin Belg. 2007;62(6):426-32. https://doi.org/10.1179/acb.2007.062.

14. Treiber KA, Lyketsos CG, Corcoran C, et al. Vascular factors and risk for neuropsychiatric symptoms in Alzheimer's disease: the Cache County Study. Int Psychogeriatr. 2008;20(3):538-53. https ://doi.org/10.1017/S1041610208006704.

15. Vik-Mo AO, Bencze J, Ballard C, Hortobágyi T, Aarsland D. Advanced cerebral amyloid angiopathy and small vessel disease are associated with psychosis in Alzheimer's disease. J Neurol Neurosurg Psychiatr. 2018;1:1.

16. McKeith IG, Dickson DW, Lowe J, et al. Diagnosis and management of dementia with Lewy bodies: third report of the DLB Consortium. Neurology. 2005;65(12):1863-72. https://doi. org/10.1212/01.wnl.0000187889.17253.b1.

17. Murray PS, Kumar S, Demichele-Sweet MA, Sweet RA. Psychosis in Alzheimer's disease. Biol Psychiat. 2014;75(7):542-52. https://doi.org/10.1016/j.biopsych.2013.08.020.

18. Livingston G, Sommerlad A, Orgeta V, et al. Dementia prevention, intervention, and care. Lancet. 2017;390(10113):2673-734. https ://doi.org/10.1016/S0140-6736(17)31363-6.

19. Lopez OL, Becker JT, Chang YF, et al. The long-term effects of conventional and atypical antipsychotics in patients with probable Alzheimer's disease. Am J Psychiatr. 2013;170(9):1051-8. https ://doi.org/10.1176/appi.ajp.2013.12081046.

20. Fernandes AC, Cloete D, Broadbent MT, et al. Development and evaluation of a de-identification procedure for a case register sourced from mental health electronic records. BMC Med Inform Decis Mak. 2013;13:71. https://doi.org/10.1186/1472-6947-13-71.

21. Perera G, Broadbent M, Callard F, et al. Cohort profile of the South London and Maudsley NHS Foundation Trust Biomedical Research Centre (SLaM BRC) Case Register: current status and recent enhancement of an Electronic Mental Health Recordderived data resource. BMJ Open. 2016;6(3):e008721. https://doi. org/10.1136/bmjopen-2015-008721.

22. Cunningham H. GATE, a general architecture for text engineering. Comput Hum. 2002;36:223-54.

23. Folstein MF, Folstein SE, McHugh PR. "Mini-mental state": a practical method for grading the cognitive state of patients for the clinician. J Psychiatr Res. 1975;12(3):189-98.

24. NHS Digital. Hospital Episode Statistics (HES) http://conte nt.digital.nhs.uk/hes. Accessed 3rd March 2017.

25. Burns A, Beevor A, Lelliott $P$, et al. Health of the Nation Outcome Scales for elderly people (HoNOS 65+). Br J Psychiatry. 1999;174:424-7.

26. Mueller C, Perera G, Hayes RD, Shetty H, Stewart R. Associations of acetylcholinesterase inhibitor treatment with reduced mortality in Alzheimer's disease: a retrospective survival analysis. Age Ageing. 2017;1:1-7. https://doi.org/10.1093/ageing/afx098.

27. Mueller C, Huntley J, Stubbs B, et al. Associations of Neuropsychiatric Symptoms and Antidepressant Prescription with Survival in Alzheimer's Disease. J Am Med Dir Assoc. 2017;18(12):107681. https://doi.org/10.1016/j.jamda.2017.07.001.

28. World Health Organisation. International Statistical Classifications of Diseases and Related Health Problems. 10th Revision Vol 2 Instruction Manual. Geneva: World Health Organisation; 2010.

29. Kadra G, Stewart R, Shetty H, et al. Extracting antipsychotic polypharmacy data from electronic health records: developing and evaluating a novel process. BMC Psychiatry. 2015;15:166. https ://doi.org/10.1186/s12888-015-0557-z.

30. Sultana J, Chang CK, Hayes RD, et al. Associations between risk of mortality and atypical antipsychotic use in vascular dementia: a clinical cohort study. Int J Geriatr Psychiatry. 2014;29(12):1249-54. https://doi.org/10.1002/gps.4101.

31. Joint Formulary Committee. British National Formulary. 69th ed. London: BMJ Group and Pharmaceutical Press; 2015.

32. Morris S, Ramsay AIG, Boaden RJ, et al. Impact and sustainability of centralising acute stroke services in English metropolitan areas: retrospective analysis of hospital episode statistics and stroke national audit data. BMJ. 2019;364:11. https://doi. org/10.1136/bmj.11.

33. Barer D, Cassidy T. Effects of diagnostic uncertainty and misclassification on hospital performance indicators for acute stroke care. Clin Med (Lond). 2014;14(6):597-600. https://doi. org/10.7861/clinmedicine.14-6-597.

34. Noble M, McLennan D, Wilkinson K, et al. The English indices of deprivation 2007;2007.

35. StataCorp LP. Stata: release 13-statistical software. TX: College Station; 2013.

36. Selvin S. Statistical analysis of epidemiologic data. New York: Oxford University Press; 1996.

37. Oudshoorn CGM, Buuren S, Rijckevorsel JLA. Flexible multiple imputation by chained equations of the AVO-95 survey: TNO Prevention and Health Leiden; 1999.

38. Rubin DB. Multiple imputation for nonresponse in surveys. Boca Raton: Wiley; 2004.

39. Lyketsos CG, Carrillo MC, Ryan JM, et al. Neuropsychiatric symptoms in Alzheimer's disease. Alzheimer's Dement J Alzheimer's Assoc. 2011;7(5):532-9. https://doi.org/10.1016/j. jalz.2011.05.2410.

40. Zhai Y, Yin S, Zhang D. Association between antipsychotic drugs and mortality in older persons with alzheimer's disease: a systematic review and meta-analysis. J Alzheimers Dis. 2016;52(2):631-9. https://doi.org/10.3233/JAD-151207.

41. Reisberg B, Borenstein J, Salob SP, Ferris SH, Franssen E, Georgotas A. Behavioral symptoms in Alzheimer's disease: phenomenology and treatment. J Clin Psychiatry. 1987;48(Suppl):9-15.

42. Degerman Gunnarsson $\mathrm{M}$, Lannfelt L, Ingelsson M, Basun H, Kilander L. High tau levels in cerebrospinal fluid predict rapid decline and increased dementia mortality in Alzheimer's disease. Dement Geriatr Cogn Disord. 2014;37(3-4):196-206. https://doi. org/10.1159/000355556.

43. Degerman Gunnarsson M, Ingelsson M, Blennow K, Basun H, Lannfelt L, Kilander L. High tau levels in cerebrospinal fluid predict nursing home placement and rapid progression in Alzheimer's disease. Alzheimers Res Ther. 2016;8(1):22. https://doi. org/10.1186/s13195-016-0191-0.

44. Bi M, Gladbach A, van Eersel J, et al. Tau exacerbates excitotoxic brain damage in an animal model of stroke. Nat Commun. 2017;8(1):473. https://doi.org/10.1038/s41467-017-00618-0.

45. Steinberg M, Lyketsos CG. Atypical antipsychotic use in patients with dementia: managing safety concerns. Am J Psychiatry. 
2012;169(9):900-6. https://doi.org/10.1176/appi.ajp.2012.12030 342.

46. Banerjee G, Carare R, Cordonnier C, et al. The increasing impact of cerebral amyloid angiopathy: essential new insights for clinical practice. J Neurol Neurosurg Psychiatry. 2017;88(11):982-94. https://doi.org/10.1136/jnnp-2016-314697.

47. Toledo JB, Cairns NJ, Da X, et al. Clinical and multimodal biomarker correlates of ADNI neuropathological findings. Acta Neuropathol Commun. 2013;1:65. https://doi. org/10.1186/2051-5960-1-65.

48. Ballard CG, Jacoby R, Del Ser T, et al. Neuropathological substrates of psychiatric symptoms in prospectively studied patients with autopsy-confirmed dementia with lewy bodies. Am J Psychiatry. 2004;161(5):843-9. https://doi.org/10.1176/appi. ajp.161.5.843.

49. Tsuang D, Simpson K, Larson EB, et al. Predicting lewy body pathology in a community-based sample with clinical diagnosis of Alzheimer's disease. J Geriatr Psychiatry Neurol. 2006;19(4):195-201. https://doi.org/10.1177/0891988706292755.

50. Mueller C, Ballard C, Corbett A, Aarsland D. The prognosis of dementia with Lewy bodies. Lancet Neurol. 2017;16(5):390-8. https://doi.org/10.1016/S1474-4422(17)30074-1.

51. Jeste DV, Finkel SI. Psychosis of Alzheimer's disease and related dementias: diagnostic criteria for a distinct syndrome. Am J Geriatr Psychiatry. 2000;8(1):29-34. https://doi.org/10.1097/00019 442-200002000-00004.

52. Cummings J, Mintzer J, Brodaty $\mathrm{H}$, et al. Agitation in cognitive disorders: international Psychogeriatric Association provisional consensus clinical and research definition. Int Psychogeriatr. 2015;27(1):7-17. https://doi.org/10.1017/S1041610214001963.

53. Bruen PD, McGeown WJ, Shanks MF, Venneri A. Neuroanatomical correlates of neuropsychiatric symptoms in Alzheimer's disease. Brain. 2008;131(Pt 9):2455-63. https://doi.org/10.1093/ brain/awn151.

54. Liu KY, Stringer AE, Reeves SJ, Howard RJ. The neurochemistry of agitation in Alzheimer's disease: a systematic review. Ageing research reviews. 2018;43:99-107. https://doi.org/10.1016/j. arr.2018.03.003.

55. Sultana J, Giorgianni F, Rea F, et al. All-cause mortality and antipsychotic use among elderly persons with high baseline cardiovascular and cerebrovascular risk: a multi-center retrospective cohort study in Italy. Expert Opin Drug Metab Toxicol. 2019;15(2):179_ 88. https://doi.org/10.1080/17425255.2019.1561860.

56. Maust DT, Kim HM, Seyfried LS, et al. Antipsychotics, other psychotropics, and the risk of death in patients with dementia: number needed to harm. JAMA Psychiatr. 2015;72(5):438-45. https://doi.org/10.1001/jamapsychiatry.2014.3018.
57. Schwertner E, Secnik J, Garcia-Ptacek S, et al. Antipsychotic treatment associated with increased mortality risk in patients with dementia: a registry-based observational cohort study. J Am Med Dir Assoc. 2019;20(3):323. https://doi.org/10.1016/j.jamda .2018.12.019.

58. Corbett A, Burns A, Ballard C. Don't use antipsychotics routinely to treat agitation and aggression in people with dementia. BMJ. 2014;349:g6420. https://doi.org/10.1136/bmj.g6420.

59. Sultzer DL, Davis SM, Tariot PN, et al. Clinical symptom responses to atypical antipsychotic medications in Alzheimer's disease: phase 1 outcomes from the CATIE-AD effectiveness trial. Am J Psychiatry. 2008;165(7):844-54. https://doi.org/10.1176/ appi.ajp.2008.07111779.

60. Schneider-Thoma J, Efthimiou O, Huhn M, et al. Second-generation antipsychotic drugs and short-term mortality: a systematic review and meta-analysis of placebo-controlled randomised controlled trials. Lancet Psychiatr. 2018. https://doi.org/10.1016/ S2215-0366(18)30177-9.

61. Ballard C, Orrell M, YongZhong S, et al. Impact of antipsychotic review and nonpharmacological intervention on antipsychotic use, neuropsychiatric symptoms, and mortality in people with dementia living in nursing homes: a factorial cluster-randomized controlled trial by the well-being and health for people with dementia (WHELD) program. Am J Psychiatr. 2016;173(3):252-62. https ://doi.org/10.1176/appi.ajp.2015.15010130.

62. McCormick N, Bhole V, Lacaille D, Avina-Zubieta JA. Validity of diagnostic codes for acute stroke in administrative databases: a systematic review. PLoS One. 2015;10(8):e0135834. https://doi. org/10.1371/journal.pone.0135834.

63. Wang T, Oliver DAP, Msosa YJ, et al. Implementation of a realtime psychosis risk detection and alerting system based on electronic health records using CogStack. J Visual Experim. 2020;1:1.

64. Morrison AP, Law H, Carter L, et al. Antipsychotic drugs versus cognitive behavioural therapy versus a combination of both in people with psychosis: a randomised controlled pilot and feasibility study. Lancet Psychiatr. 2018. https://doi.org/10.1016/S2215 -0366(18)30096-8.

65. Brodaty H, Arasaratnam C. Meta-analysis of nonpharmacological interventions for neuropsychiatric symptoms of dementia. Am J Psychiatry. 2012;169(9):946-53. https://doi.org/10.1176/appi. ajp.2012.11101529.

Publisher's Note Springer Nature remains neutral with regard to jurisdictional claims in published maps and institutional affiliations. 